Original paper

\title{
Pediatric chronic liver failure-sequential organ failure assessment score and outcome of acute liver failure in children
}

\author{
Bassam Abdel Hakam Ayoub, Mohammed Abdel Hafez Ali, Tahany Abdel Hamid Salem, Marwa Sabry Rizk, \\ Salma Abdel Megeed Nagi, Nermin Mohammed Adawy \\ National Liver Institute - Menoufia University, Egypt
}

\begin{abstract}
Aim of the study: Liver transplantation remains the only definitive treatment for children with acute liver failure proven to have irreversible liver injury. Many prognostic models have been used for outcome prediction in pediatric acute liver failure to select patients in a real need of liver transplantation, but unfortunately all have shown inconsistent reproducibility and prognostic accuracy. The aim of this study was to evaluate the pediatric chronic liver failure sequential organ failure assessment (pCLIF-SOFA) score as a predictor of pediatric acute liver failure outcome.

Material and methods: Clinical and laboratory data of 41 children with acute liver failure admitted to the National Liver Institute - Menoufia University were collected retrospectively and used for calculation of both PCLIF-SOFA and Pediatric End-Stage Liver Disease (PELD)/Model for End-Stage Liver Disease (MELD) scores on the day of admission, then statistical analysis was performed to identify the ability of these scores to predict the outcome.

Results: According to the outcome, children enrolled in this study were allocated to survived $(n=16)$ and died $(n=25)$ groups, which were age and sex matched. The non-survival group had significantly higher values of both pCLIF-SOFA score (11 [7-13]) and PELD/MELD score (36 [18-42]) than those of the survival group (8 [7-11], 27.5 [15-45]; $p<0.001, p=0.004$ ) respectively. Both pCLIF-SOFA and PELD/MELD scores at cut-off values $>8$ and $>30$ respectively on admission could predict death in children with acute liver failure (ALF) with high sensitivity, but with higher specificity, positive and negative predictive values for pCLIF-SOFA.

Conclusions: pCLIF-SOFA is a good predictor of death in pediatric acute liver failure.
\end{abstract}

Key words: children, liver failure, organ failure, outcome.

Address for correspondence:

Bassam Abdel Hakam Ayoub, Department of Pediatric Hepatology, Gastroenterology and Nutrition, National Liver Institute, Menoufia University, 32511 Shebin El-koom, Menoufia, Egypt, phone: +2-048-222-2740, fax: +2-048-223-4586,

e-mail: bassamayob@yahoo.com

\section{Introduction}

Acute liver failure (ALF) in children is a dramatic clinical syndrome characterized by deterioration in liver function tests, and potentially associated with dysfunction in other organs $[1,2]$. Although rare, ALF is a potentially devastating process that often needs urgent liver transplantation (LT) when liver regeneration is unlikely [3]. There are a large variety of causes of ALF, and its evolution and rate of progression are very heterogeneous, complicating the defi- nition of its reversibility and of the moment of need for LT [4].

Despite new therapies and support measures, survival continues to be unsatisfactory, ranging between $10 \%$ and $40 \%$ [5]. After the introduction of LT as a therapeutic option for patients with ALF, the survival rate reached $60 \%$ to $80 \%$ [6]. Major benefits provided by LT are limited by its relatively low applicability, either due to development of contraindications such as irreversible brain damage or multi-organ failure or the unavailability of an organ donor in a timely fashion [7]. 
When to list a child is critical for the success of LT, knowledge of sensitive prognostic markers could also determine whether there is a possibility of recovery without LT. Transplantation implies surgical risks and permanent immunosuppression in a patient with a potentially reversible disease. LT must be indicated whenever chances for survival are lower without rather than with transplantation [8].

Numerous prognostic models have been investigated mainly in adults with varying success. King's College criteria (KCC) were the first and are the standard upon which others are judged. KCC take into account patient demographics that include diagnosis and age, degree of clinical encephalopathy, as well as biochemical determination of coagulopathy, arterial $\mathrm{pH}$, serum bilirubin, and creatinine [9]. Several other models have been developed and include the use of serum lactate [10], $\alpha$-fetoprotein [11], serum albumin, lactate, valine, and pyruvate [12], serum phosphate [13] and Model for End-Stage Liver Disease (MELD), which was originally developed to estimate post-procedural mortality in cirrhotic patients undergoing transjugular intrahepatic porto-systemic shunts (TIPS) [7].

The ideal prognostic model remains elusive, as none of the currently used models could perform extremely well [14].

The aim of the present study was to investigate the prognostic accuracy of the pediatric chronic liver failure sequential organ failure assessment (pCLIF-SOFA) score in comparison to the Pediatric End-Stage Liver Disease (PELD)/MELD score in children with ALF admitted to our tertiary center.

\section{Material and methods}

Forty-one children (22 males and 19 females, age: $0.2-16$ years), admitted to the National Liver Institute - Menoufia University between September 2016 and September 2018 and fulfilling the criteria of the Pediatric Acute Liver Failure (PALF) Study Group definition of ALF [15], were enrolled retrospectively from the patients files.

All patients' data were collected including: detailed history, clinical findings (including vital signs, partial pressure of arterial oxygen $\left(\mathrm{PaO}_{2}\right)$ and fraction of inspired oxygen $\left(\mathrm{FiO}_{2}\right)$ ), laboratory tests (total bilirubin (TB), direct bilirubin (DB), alanine transaminase (ALT), aspartate transaminase (AST), serum albumin, international normalized ratio (INR), blood ammonia, serum creatinine and complete blood count), abdominal ultrasonographic findings, final diagnosis and outcome of cases.
According to patients' outcome they were allocated into two groups: the survival group $(n=16)$ and non-survival group $(n=25)$.

Using patients' data of the first day of admission, pCLIF-SOFA score [16], PELD score (for children $<12$ years old) [17] and MELD score (for children $\geq 12$ years old) $[18,19]$ were calculated.

\section{Statistical analysis}

Data were collected and entered into the computer using the SPSS program for statistical analysis (version 21). Data were entered as numerical or categorical, as appropriate. The Kolmogorov-Smirnov test of normality revealed significance in the distribution of most of the variables, so the non-parametric statistics was adopted. Data were described using minimum, maximum and median. Categorical variables were described using frequency and percentage. Comparisons were carried out between two studied independent non-normally distributed subgroups using the Mann-Whitney $U$ test. The $\chi^{2}$ test was used to test the association between qualitative variables. MedCalc Software version 14 was used to draw receiver operating characteristic (ROC) curves to detect the discriminative performance of each of PCLIF-SOFA and PELD/MELD scores by calculating the area under the curve (AUC), where AUC above 0.7 is considered fair while a value between 0.8 and 0.9 and above 0.9 indicated good and excellent diagnostic accuracy respectively. The same program was used also to compare the two ROC curves to test the statistical significance of the difference between the areas under ROC curves. Sensitivity, specificity, positive predictive value (PPV) and negative predictive value (NPV) were expressed as percentages. Results were considered significant if the $p$ value $<0.05$.

\section{Results}

The most common causes of ALF in the study groups were hepatitis A and indeterminate hepatitis (Fig. 1).

There was no statistically significant difference between survival and non-survival groups as regard age, sex, time interval between onset of jaundice and liver failure, hepatomegaly, splenomegaly or presence of ascites. Also, there was no statistically significant difference between the two groups as regard serum TB, AST, ALT, albumin, creatinine, blood ammonia, hemoglobin $(\mathrm{Hb})$, platelet count or white blood cell (WBC) count. The survival was not statistically different be- 


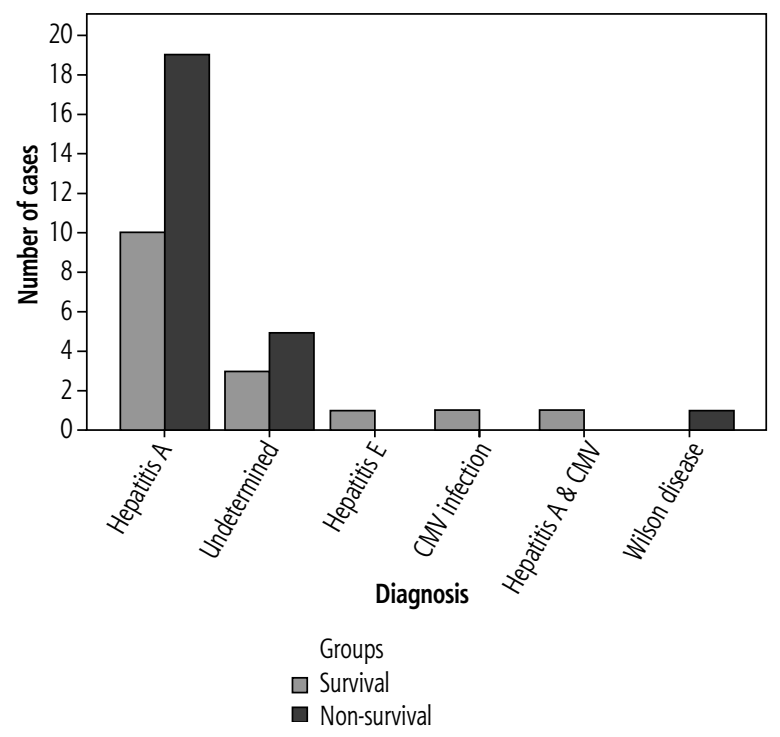

tween the hepatitis A group and the group of other diagnoses (Table 1).

The frequency of hepatic encephalopathy grade III-IV on the first day of admission was significantly higher in the non-survival group than in the survival group $(p=0.001)$. Also INR of the non-survival group on admission (4.4 [1.80-10.3]) was significantly higher than that of the survival group (2.6 [1.70-8.2]) $(p=0.02)$ (Table 1).

Only 3 cases of the non-survival group had $\mathrm{PaO}_{2} /$ $\mathrm{FiO}_{2}$ ratio < 400 but $>300$; otherwise all cases of both groups had a ratio $>400$. Also, there was no hypotension except in 3 cases in the non-survival group but with no need of circulatory support with dopamine or epinephrine, so there was no statistically significant difference between the two groups as regards the two parameters $(p=0.150)$

Fig. 1. Diagnoses of the study groups

Table 1. Demographic, clinical, ultrasonographic and laboratory characteristics of survival and non-survival groups

\begin{tabular}{|c|c|c|c|}
\hline Parameter & Survival $(n=16)$ & Non-survival $(n=25)$ & $P$-value \\
\hline Age (years) & $3.5(0.2-15)$ & $4(1-16)$ & 0.361 \\
\hline \multicolumn{4}{|l|}{ Sex, $n(\%)$} \\
\hline Male & $8(50)$ & $14(56)$ & \multirow[t]{2}{*}{0.707} \\
\hline Female & $8(50)$ & $11(44)$ & \\
\hline Ascites, $n(\%)$ & $3(18.8)$ & $10(40)$ & 0.154 \\
\hline Hepatomegaly, $n(\%)$ & $9(56.3)$ & $16(64)$ & 0.620 \\
\hline Splenomegaly, $n(\%)$ & $8(50)$ & $10(40)$ & 0.529 \\
\hline Jaundice/failure interval (days) & $18(10-35)$ & $15(4-50)$ & 0.452 \\
\hline \multicolumn{4}{|l|}{ Hepatic encephalopathy, $n(\%)$} \\
\hline Grade I-II & $5(31.3)$ & $6(24)$ & \multirow[t]{3}{*}{$0.001^{*}$} \\
\hline Grade III-IV & $1(6.3)$ & $15(60)$ & \\
\hline No encephalopathy & $10(62.5)$ & $4(16)$ & \\
\hline $\mathrm{TB}(\mathrm{mg} / \mathrm{dl})$ & $23.3(6.6-36.8)$ & $22.6(5-52)$ & 0.915 \\
\hline AST (IU/I) & $1031(62-2978)$ & 699 (58-4565) & 0.487 \\
\hline ALT (IU/I) & $597.5(59-2993)$ & $993(47-4648)$ & 0.513 \\
\hline INR & $2.6(1.70-8.2)$ & $4.4(1.8-10.3)$ & $0.02^{*}$ \\
\hline $\mathrm{ALB}(\mathrm{gm} / \mathrm{dl})$ & $3.7(1.3-4.3)$ & $3.2(2.3-4.7)$ & 0.068 \\
\hline Blood ammonia ( $\mu \mathrm{mol} / \mathrm{l})$ & $216(104-452)$ & $220(95-617)$ & 0.789 \\
\hline Creatinine (mg/dl) & $0.4(0.16-0.8)$ & $0.5(0.2-5.2)$ & 0.167 \\
\hline $\mathrm{Hb}(\mathrm{gm} / \mathrm{dl})$ & $9.5(6.6-16.1)$ & $10.9(3.5-14.6)$ & 0.521 \\
\hline WBCs $\left(\times 10^{3} / \mathrm{dl}\right)$ & $9.7(3.3-26)$. & $10.9(3.40-25)$ & 0.446 \\
\hline Platelets (× 103/dl) & $316(79-580)$ & $239(34-739)$ & 0.209 \\
\hline \multicolumn{4}{|l|}{ Diagnosis } \\
\hline Hepatitis A, $n(\%)$ & $10(34.5)$ & $19(65.5)$ & \multirow[t]{2}{*}{0.354} \\
\hline Other diagnoses, $n(\%)$ & $6(50)$ & $6(50)$ & \\
\hline
\end{tabular}


A

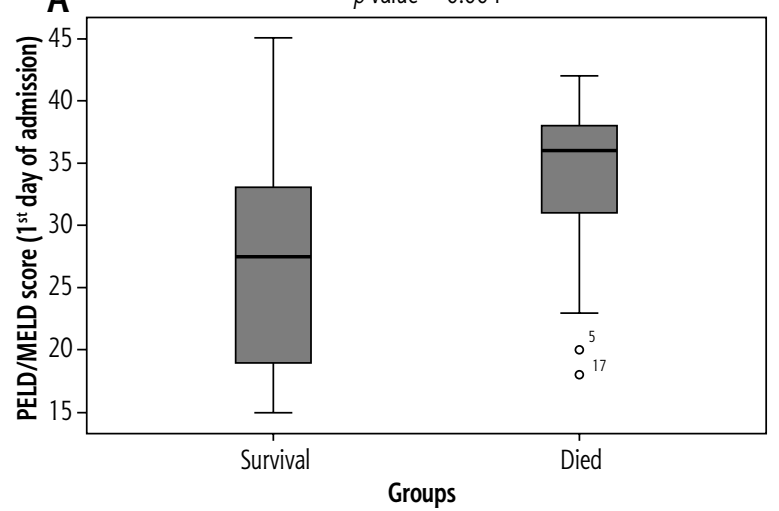

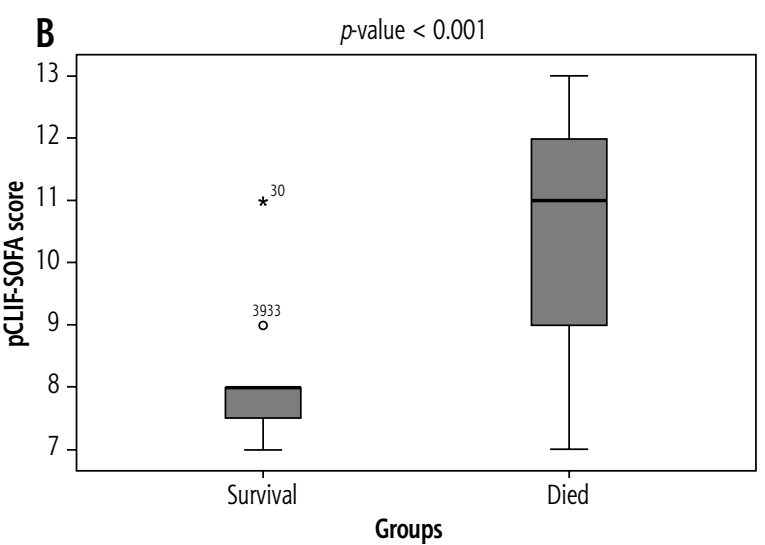

Fig. 2. Comparison of both MELD/PELD score (A) and PCLLF-SOFA score (B) between the study groups

On admission, pCLIF-SOFA score of the non-survival group (11 [7-13]) was significantly higher than that of the survival group $(8[7-11])(p<0.001)$. Also, the non-survival group had a statistically significantly higher PELD/MELD score (36 [18-42]) than that of the survival group (27.5 [15-45]) ( $p=0.004)$ (Fig. 2).

Both pCLIF-SOFA and PELD/MELD scores, on admission, at cut-off values $>8$ and $>30$, respectively, could predict death in children with ALF with higher AUC for pCLIF-SOFA score (0.88, 95\% CI: 0.74-0.96, $p<0.001)$ than that of PELD/MELD score $(0.76$, 95\% CI: $0.61-0.88, p=0.001)$, but this difference was not statistically significant $(p=0.181)$. Both scores have the same sensitivity (84\%) but with higher specificity, PPV and NPV for PCLIF-SOFA score $(81 \%, 88 \%$ and $76 \%$ respectively) than those of PELD/MELD score (63\%, $78 \%$ and $71 \%$ respectively) (Fig. 3 ).

The majority of cases in the current study were diagnosed as acute hepatitis $\mathrm{A}$, so we compared both pCLIF-SOFA and PELD/MELD scores between survival and non-survival cases in this group. On admission, pCLIF-SOFA score of non-surviving acute hepatitis A patients (11 [8-13]) was significantly higher than that of surviving ones $(8[7-11])(p<0.001)$. Also, non-surviving patients had a statistically significant higher PELD/MELD score (36 [28-42]) than that of surviving patients $(23.5[15-34])(p<0.001)$ (Fig. 4).

\section{Discussion}

Pediatric ALF is a potentially devastating condition which occurs in previously healthy children and frequently leads to rapid clinical deterioration [20]. It is well established, in both adults and children, that ALF is a complex multiorgan illness, and that mortality increases with severe sepsis and multiorgan failure [21].

Liver transplantation remains the only definitive treatment for patients with ALF proven to have irre-

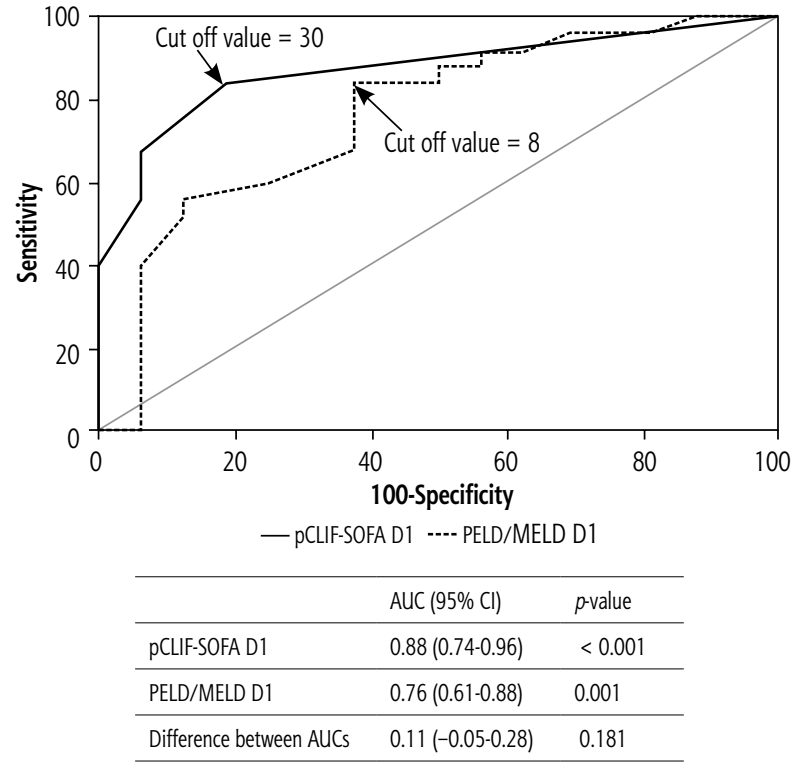

pCLIF-SOFA - pediatric chronic liver failure sequential organ failure assessment, PELD - Pediatric EndStage Liver Disease, MELD - Model for End-Stage Liver Disease, AUC - area under curve,

$\mathrm{Cl}$ - confidence interval, D1 - day one of admission

Fig. 3. Receiver operating characteristic curve showing the discrimination ability of the PCLIF-SOFA and PELD/MELD score in predicting death in children with ALF in our study

versible liver injury. A number of prognostic models have been used for outcome prediction in ALF patients to select patients in need of LT. These models have shown inconsistent reproducibility and prognostic accuracy and therefore cannot be taken to reliably predict mortality in ALF [22].

So, there is an urgent need for dynamic prognostic scoring systems for PALF with potential inclusion of physiological and inflammatory parameters. However, determining the prognosis remains challenging [23].

In this retrospective study we studied 41 children admitted to the National Liver Institute - Menoufiya University and fulfilling the criteria of the PALF Study 
A

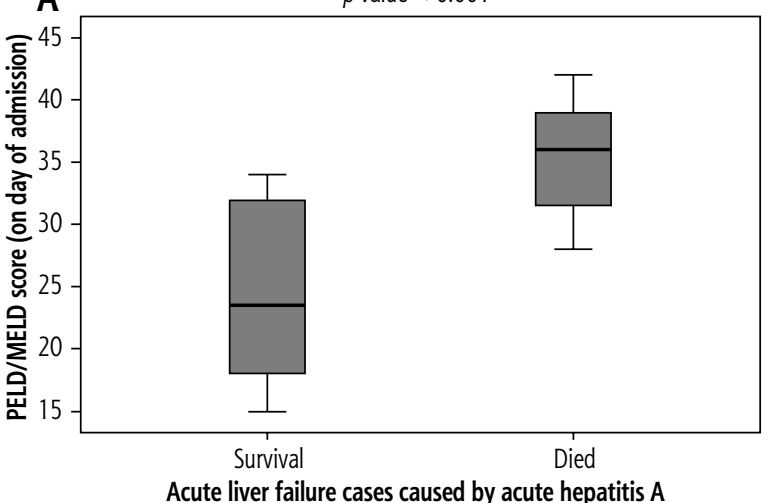

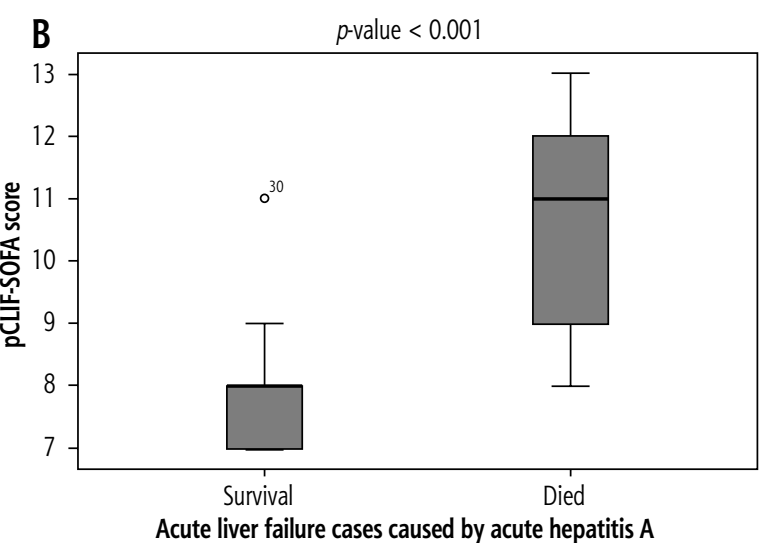

Fig. 4. Comparison of both MELD/PELD score (A) and pCLIF-SOFA score (B) between survival and non-survival cases of acute liver failure caused by acute hepatitis $A$

Group definition of ALF to evaluate pCLIF-SOFA as a predictor of PALF outcome in comparison with PELD/MELD score.

According to outcome, children in the current study were allocated to two, age- and sex-matched, groups: the survival group $(n=16)$ and the non-survival group $(n=25)$.

The most common causes of ALF in the study groups were hepatitis $\mathrm{A}$ and indeterminate hepatitis. Hepatitis A virus is a common cause of PALF in areas where it is endemic $[24,25]$, while indeterminate hepatitis represents approximately $30 \%$ of PALF cases and in patients aged 1-5 years this percentage exceeds $60 \%[26]$.

There was no statistically significant difference between survival and non-survival groups as regards diagnosis, time interval between onset of jaundice and liver failure, hepatomegaly, splenomegaly and presence of ascites. Also, there was no statistically significant difference between the two groups as regards serum TB, AST, ALT, albumin, creatinine, blood ammonia, $\mathrm{Hb}$, platelet count and WBC count.

On the other hand, we found that the frequency of hepatic encephalopathy grade III-IV on the first day of admission was significantly higher in the non-survival group than in the survival group. The severity of HE in ALF is a poor prognostic marker, and the longer the duration of severe encephalopathy (grade III or IV) before LT, the worse the prognosis after LT [27].

In our study we found that INR of the non-survival group on admission was significantly higher than that of the survival group. This is in agreement with Özçay et al., who found in their study on children with ALF that survivors had a significantly lower INR than that of non-survivors [28]. But this contrasts with the findings of Kaur et al., who reported in a study on 43 children with ALF that INR cannot be used as a prognostic factor for ALF outcome [29].
In an attempt to improve current prognostic scores, the efficacy of established intensive care unit (ICU) predictive models was studied, to grade PALF severity. Mixed results are available when applying these models to ALF [23].

In our study we found, on admission, that the non-survival group had statistically significant higher values of both pCLIF-SOFA and PELD/MELD scores than those of the survival group, which was the same finding among the cases of acute hepatitis A separately. Our result is in agreement with many studies which proved that children with ALF who died or underwent LT had a statistically significantly higher mean PELD/ MELD score than that of those who survived without LT [7, 30, 31], while Chien et al. reported that PELD/ MELD scores' associations with outcome were not significant [32].

While sequential organ failure assessment (SOFA) score outperformed the other prognostic scores (King's College criteria and MELD score) in predicting outcome of acetaminophen-induced ALF in adults [33], pCLIF-SOFA, developed for the first time by Bolia et al. for mortality prediction in children with decompensated chronic liver disease [16], was not investigated before as a predictor of PALF outcome except in our study.

In our study we found that both pCLIF-SOFA and PELD/MELD scores $>8$ and $>30$ respectively on admission could predict death in children with ALF with the same sensitivity but with higher specificity, PPV, NPV and AUC for pCLIF-SOFA score. Sanchez and D'Agostino reported that PELD/MELD score at a cutoff value of 33 can predict poor prognosis with a specificity of $81 \%$ and a sensitivity of $86 \%$ [34]. Also Rajanayagam et al. found that PELD/MELD score at cutoffs $>27$ and $>42$ can predict death with sensitivity of $76 \%$ and $66 \%$ and specificity of $60 \%$ and $92 \%$, respectively [35]. 
This outperformance of the pCLIF-SOFA score seems to be due to giving points to grade of encephalopathy, which is not done in the PELD/MELD score.

The strength of our study is the reasonable sample size, while its limitation is unavailability of the follow-up data of many of patients, which limited the dynamic application of the PCLIF-SOFA score.

In conclusion, the pCLIF-SOFA score is better than the PELD/MELD score as a predictor of death in PALF and can be used for accurate selection of children with ALF who are in a real need of LT. But this should be validated through future large scale prospective studies.

\section{Disclosure}

The authors declare no conflict of interest.

\section{References}

1. Mendizabal M, Silva MO. Liver transplantation in acute liver failure: A challenging scenario. World J Gastroenterol 2016; 22: 1523-1531.

2. Wendon J, Cordoba J, Dhawan A, et al. EASL clinical practical guidelines on the management of acute (fulminant) liver failure. J Hepatol 2017; 66: 1047-1081.

3. Farmer DG, Venick RS, McDiarmid SV, et al. Fulminant hepatic failure in children: superior and durable outcomes with liver transplantation over 25 years at a single center. Ann Surg 2009; 250: 484-493.

4. Devictor D, Tissieres P, Durand P, et al. Acute liver failure in neonates, infants and children. Expert Rev Gastroenterol Hepatol 2011; 5: 717-729.

5. Sundaram SS, Alonso EM, Narkewicz MR, et al. Characterization and outcomes of young infants with acute liver failure. J Pediatr 2011; 159: 813-818.e1.

6. Rhee C, Narsinh K, Venick RS, et al. Predictors of clinical outcome in children undergoing orthotopic liver transplantation for acute and chronic liver disease. Liver Transpl 2006; 12: 13471356.

7. Yantorno SE, Kremers WK, Ruf AE, et al. MELD is superior to King's College and Clichy's criteria to assess prognosis in fulminant hepatic failure. Liver Transpl 2007; 13: 822-828.

8. Dhiman RK, Jain S, Maheshwari U, et al. Early indicators of prognosis in fulminant hepatic failure: an assessment of the Model for End-Stage Liver Disease (MELD) and King's College Hospital criteria. Liver Transpl 2007; 13: 814-821.

9. Sundaram V, Shneider BL, Dhawan A, et al. King's College Hospital Criteria for non-acetaminophen induced acute liver failure in an international cohort of children. J Pediatr 2013; 162 319-323.

10. Hadem J, Strassburg CP, Manns MP. Prediction of outcome and selection of the liver transplantat candidate in acute liver failure. Front Physiol 2012; 3: 340.

11. Ashry S, Ahmed S, Salem H, et al. Alpha fetoprotein: a prognostic marker for early detection of liver regeneration in acute paracetamol toxicity. QJM 2018; 111 (Suppl 1).

12. Dabos KJ, Newsome PN, Parkinson JA, et al. A biochemical prognostic model of outcome in paracetamol-induced acute liver injury. Transplantation 2005; 80: 1712-1717.
13. Schmidt LE, Dalhoff K. Serum phosphate is an early predictor of outcome in severe acetaminophen-induced hepatotoxicity. Hepatology 2002; 36: 659-665.

14. O'Grady J. Timing and benefit of liver transplantation in acute liver failure. J Hepatol 2014; 60: 663-670.

15. Squires Jr RH, Shneider BL, Bucuvalas J, et al. Acute liver failure in children: the first 348 patients in the pediatric acute liver failure study group. J Pediatr 2006; 148: 652-658.e2.

16. Bolia R, Srivastava A, Yachha SK, et al. Pediatric CLIF-SOFA score is the best predictor of 28-day mortality in children with decompensated chronic liver disease. J Hepatol 2018; 68: 449-455.

17. Wiesner RH, McDiarmid SV, Kamath PS, et al. MELD and PELD: application of survival models to liver allocation. Liver Transpl 2001; 7: 567-580.

18. Malinchoc M, Kamath PS, Gordon FD, et al. A model to predict poor survival in patients undergoing transjugular intrahepatic portosystemic shunts. Hepatology 2000; 31: 864-871.

19. Wiesner R, Edwards E, Freeman R, et al. Model for end-stage liver disease (MELD) and allocation of donor livers. Gastroenterology 2003; 124: 91-96.

20. Alonso EM, Horslen SP, Behrens EM, et al. Pediatric acute liver failure of undetermined cause: A research workshop. Hepatology 2017; 65: 1026-1037.

21. Kamath PS, Heimbach J, Wiesner RH. Acute liver failure prognostic scores: is good enough good enough? Clin Gastroenterol Hepatol 2016; 14: 621-623.

22. Pelaez-Luna M, Martinez-Salgado J, Olivera-Martinez MA. Utility of the MAYO End-Stage Liver Disease score, King's College Criteria, and a new in-hospital mortality score in the prognosis of in-hospital mortality in acute liver failure. Transplant Proc 2006; 38: 927-929.

23. Jain V, Dhawan A. Prognostic modeling in pediatric acute liver failure. Liver Transpl 2016; 22: 1418-1430.

24. Lee WS, McKiernan P, Kelly DA. Etiology, outcome and prognostic indicators of childhood fulminant hepatic failure in the United Kingdom. J Pediatr Gastroenterol Nutr 2005; 40: 575-581.

25. Turk T, Al Saadi T, Sawaf B, et al. Progressive liver failure post acute hepatitis A, over a three-month period, resulting in hepatorenal syndrome and death. Gastroenterol Rep 2017; 5: 161-164.

26. Narkewicz MR, Dell Olio D, Karpen SJ, et al. Pattern of diagnostic evaluation for the causes of pediatric acute liver failure: an opportunity for quality improvement. J Pediatr 2009; 155: 801-806.e1.

27. Novelli G, Rossi M, Morabito V, et al. Pediatric acute liver failure with molecular adsorbent recirculating system treatment. Transplant Proc 2008; 40: 1921-1924.

28. Özçay F, Karadağ-Öncel E, Bariş Z, et al. Etiologies, outcomes, and prognostic factors of pediatric acute liver failure: A single center's experience in Turkey. Turk J Gastroenterol 2016; 27: 450-457.

29. Kaur S, Kumar P, Kumar V, et al. Etiology and prognostic factors of acute liver failure in children. Indian Pediatr 2013; 50: 677-679.

30. Sanchez MC, D’Agostino DE. Pediatric end-stage liver disease score in acute liver failure to assess poor prognosis. J Pediatr Gastroenterol Nutr 2012; 54: 193-196.

31. Wiesner RH. MELD/PELD and the allocation of deceased donor livers for status 1 recipients with acute fulminant hepatic failure, primary nonfunction, hepatic artery thrombosis, and acute Wilson's disease. Liver Transpl 2004; 10: 17-22.

32. Chien MM, Chang MH, Chang KC, et al. Prognostic parameters of pediatric acute liver failure and the role of plasma exchange. Pediatr Neonatol 2019; 60: 389-395. 
33. Cholongitas E, Theocharidou E, Vasianopoulou P, et al. Comparison of the sequential organ failure assessment score with the King's College Hospital criteria and the model for end-stage liver disease score for the prognosis of acetaminophen-induced acute liver failure. Liver Transpl 2012; 18: 405-412.

34. Sanchez MC, D’Agostino DE. Pediatric end-stage liver disease score in acute liver failure to assess poor prognosis. J Pediatr Gastroenterol Nutr 2012; 54: 193-196.

35. Rajanayagam J, Coman D, Cartwright D, et al. Pediatric acute liver failure: etiology, outcomes, and the role of serial pediatric end-stage liver disease scores. Pediatr Transplant 2013; 17: 362-368. 